Gert-Joachim Glaeßner

\title{
Demokratie nach dem Ende des Kommunismus
}

Antrittsvorlesung

21. Juni 1993

Humboldt-Universität zu Berlin

Fachbereich Sozialwissenschaften 
Herausgeberin:

Die Präsidentin der Humboldt-Universität zu Berlin

Prof. Dr. Marlis Dürkop

Copyright: Alle Rechte liegen beim Verfasser.

Redaktion:

Christine Gorek

Forschungsabteilung der Humboldt-Universität

Unter den Linden 6

10099 Berlin

Herstellung:

Linie DREI, Agentur für Satz und Grafik

Wühlischstraße 33

10245 Berlin

\section{Heft 15}

Redaktionsschluß:

02. 11.1993 
Demokratie nach dem Ende des Kommunismus - hinter diesem Titel meines Vortrages verbergen sich zwei Fragen: 1. Wird am Ende des welthistorischen Umbruchs, der etwa 1985 mit Glasnost und Perestroika in der Sowjetunion begann, der 1989 in die Revolutionen in Mittel-Ost- und Südosteuropa mündete und 1991 zum Ende der einstmaligen „Vorhut der Weltrevolution“, der Sowjetunion führte, die Demokratie stehen? 2. Wie stellt sich die Zukunft der etablierten Demokratien des Westens nach dem Umbruch in den kommunistischen Ländern dar?

Beide Dimensionen sollen im folgenden angesprochen werden. Stellt man gegenwärtig die Frage nach der Zukunft der Demokratie, so ist das Bild widersprüchlich. Einerseits sind einige hoffnungsvolle Zeichen erkennbar, daß die Demokratie eine neue Chance hat. In Lateinamerika, in Brasilien, Argentinien, Paraguay, Uruguay und Chile wurden blutige Diktaturen überwunden, in Nicaragua ein zwischen marxistisch-leninistischen und nationalistisch-sozialistischen Ideen changierendes Regime durch demokratische Wahlen abgelöst und ein Bürgerkrieg, der nicht unwesentlich von außen geschürt wurde, beendet.

Auf dem afrikanischen Kontinent hat mit dem Ende des OstWest-Konflikts die Zeit der „Stellvertreterbürgerkriege“ geendet oder hat, wie in Mocambique und Angola, doch große Chancen zu einem Ende zu kommen. Namibia hat einen bemerkenswert erfolgreichen Transitionsprozeß durchgemacht. Selbst das Bollwerk des Antikommunismus und Rassismus, Südafrika hat eine realistische Chance für eine demokratische Zukunft.

In Asien hat Südkorea einen demokratischen Regimewechsel hinter sich. In vielen, von Diktaturen regierten Ländern regen sich oppositionelle demokratische Kräfte.

Und schließlich: Der Kommunismus sowjetischer Prägung, ein dreiviertel Jahrhundert lang, der Gegenpol zu liberal-demokratischen und marktwirtschaftlichen Systemen, brach binnen Kur- 
zem zusammen und hinterließ Staaten, die nahezu uni sono eine demokratische Entwicklung anstreben oder anzustreben vorgeben.

Andererseits bleibt festzuhalten, daß nur eine Minderheit der Staaten der Welt demokratisch regiert wird, daß die Mehrheit der Mitglieder der Vereinten Nationen die UN-Charta und die allgemeinen Menschenrechte nicht beachten und daß dies auch in überschaubarer Zukunft nicht zu erwarten ist.

In der Euphorie des Jahres 1989 schien es für kurze Zeit, als wäre die liberale Demokratie als ,Sieger“ aus der Systemauseinandersetzung hervorgegangen. Es verwundert nicht, daß angesichts dieser Entwicklung große Hoffnungen in eine demokratische und damit friedlichere Zukunft gesetzt wurden.

Shlomo Avineri hat von einer ,fast messianischen Hoffnung“ gesprochen - nicht nur in den Gesellschaften, die den Kommunismus überwunden hatten, sondern auch im Westen. ${ }^{1}$

Während der atemberaubenden Ereignisse Ende 1989/Anfang 1990 bezweifelten nur wenige, daß der schnelle Übergang zur Marktwirtschaft nötig und möglich und die Demokratie das gleichsam natürliche Korrelat zur Marktwirtschaft sei.

Insofern war die Überzeugung der neuen Eliten in den post-kommunistischen Ländern, die möglichst schnelle Einführung der Marktwirtschaft werde auch die Grundlagen für eine demokratische politische Ordnung schaffen, verständlich. Sie reflektierte die weithin geteilte Grundüberzeugung, daß Kapitalismus und Demokratie Hand in Hand gehen, daß die Demokratie die charakteristische politische Form des Kapitalismus sei. (Daß hierin ironischerweise eine Parallele zu Grundüberzeugungen Lenins, wenngleich mit anderer Betonung festzustellen ist, sei en passant angemerkt.) $)^{2}$ Inzwischen ist Ernüchterung eingekehrt.

Der Zerfall des Kommunismus hat nicht nur alle seit mehr als einem halben Jahrhundert bestehenden Konfrontationen beendet, er hat auch den sicheren Rahmen, in dem sich politisches Denken bewegte, zerstört.

Die alte Unterscheidung Freiheit gegen Totalitarismus, Demokratie gegen Diktatur, West gegen Ost ist hinfällig. Hinfällig ist zumindest gegenwärtig - auch die Angst vor der atomaren Apokalypse - dafür finden die regional begrenzten Ungeheuer- 
lichkeiten direkt vor unserer Tür, in Europa, und nicht mehr nur „weit hinten“ in der sogenannten Dritten Welt statt.

Nach dem Ende der Sowjetunion hat nicht nur der ideologische und politisch-militärische Gegner, sondern auch eine Ordnungsmacht die Bühne verlassen. Man muß nicht so weit gehen, wie Alain Minc, der 1990 in einem Buch über „Die Wiedergeburt des Nationalismus in Europa" ${ }^{\text {"3 }}$ bemerkte, Westeuropa habe seit 1945 in der Überzeugung gelebt, im Osten herrsche Ordnung, ja die Ordnung könne sogar aus dem Osten kommen. Die Ordnung von Jalta, deren konstitutiver Teil die Sowjetunion war, führte zu einer Situation, die Alain Minc folgendermaßen beschreibt:

„Solange die Ordnung herrschte, lebten wir mit der Bedrohung ohne Risiken. Mit dem völlig ungeordneten Zustand triumphieren die Risiken ohne eine große Bedrohung. Auf der Ebene des ,Krisenmanagements“ ist das zweite Szenarium ungleich gefährlicher als das erste. “4

Die Spätfolgen des Jahres 1989 bedeuten zum einen das Ende der Blöcke mit ihren höchst fragilen Sicherheitsstrukturen und gegenseitigen Abhängigkeiten. Zum anderen bedeuten sie aber auch das Ende von Demokratievorstellungen, die ihre Legitimation im wesentlichen aus der Abgrenzung gegenüber dem Kommunismus bezogen. Dies trifft insbesondere für Deutschland zu. Hier war trotz aller Versuche, die Blockkonfrontation zu mildern - das Selbstverständnis der beiden deutschen Teilstaaten und Teilgesellschaften in besonderer Weise von der Notwendigkeit der Abgrenzung bestimmt.

Diese Bemerkungen sollten meine Grundthese vorbereiten, daß es nach dem Ende des Kommunismus in Europa erstens um den Aufbau von Demokratien in den post-kommunistischen Ländern geht, und daß zweitens die Demokratien des Westens vor der Notwendigkeit stehen, sich ihrer eigenen Grundlagen neu zu vergewissern.

\section{Regimewechsel und Systemtransformation}

Der Kollaps des Kommunismus bedeutet mehr als den Zusammenbruch einer politischen Ordnung. In mehr als vierzig Jahren 
hatten die sozialistischen Systeme auch eine eigene ökonomische und soziale Ordnung errichtet und konsolidiert, neue kulturelle Muster verordnet und implementiert und neue soziale Strukturen und Verhaltensweisen geschaffen. Das totale Monopol des Staates, bzw. der Staatspartei auf die Gestaltung aller Sphären der Gesellschaft unterschied den Kommunismus von anderen, nicht weniger strengen Diktaturen auch dann noch, als mit dem Ende des „Hochstalinismus“ eine gewisse Öffnung des politischen Systems einsetzte. ${ }^{5}$

Anders als in zeitlich begrenzten, wenngleich folgenreichen Diktaturen wie dem Nationalsozialismus, dem italienischen Faschismus oder den autoritären Regimen in Südeuropa oder Lateinamerika, war der sowjetische Sozialismus in der Lage, über mehrere Jahrzehnte und mehrere Generationen hinweg seine ideologischen Zielvorstellungen in die Praxis umzusetzen. Dies geschah nie ohne Zwang, aber auch mit Duldung, auch mit Unterstützung aus der Bevölkerung - auch vieler derer, die 1989 aufbegehrten.

Um so mehr stellt sich die Frage nach den tieferliegenden Ursachen für den Systemkollaps. Lassen Sie mich das Problem mit einem Zitat von Octavio Paz einkreisen:

„Die moderne Demokratie“, schreibt er 1992, „wird von keinem äußeren Feind, sondern von den ihr innewohnenden Schwächen bedroht. Sie hat den Kommunismus besiegt, aber sich selbst hat sie nicht überwinden können. Ihre Schwächen sind das Ergebnis des Widerspruchs, der ihr seit ihrem Entstehen anhaftet: des Gegensatzes zwischen Freiheit und Brüderlichkeit."

Octavio Paz formuliert hier einen Grundkonflikt freiheitlicher Demokratien. Seine Aussage läßt sich aber auch umkehren: Der Kommunismus ist nicht von einem äußeren Feind besiegt worden, sondern an seinen inneren Widersprüchen zugrunde gegangen. Anspruch und Wirklichkeit klafften immer weiter auseinander. Der Geburtsfehler der kommunistischen Systeme war die Absicht, soziale Gleichheitsrechte zu schaffen, ohne individuelle Freiheitsrechte zu verwirklichen. Die politische Freiheit wurde geopfert, aber Gleichheit wurde nicht erreicht. Vielmehr wurden neue Formen der Ungleichheit produziert, vor allem in der Sphäre der Macht. 
Die Revolutionen des Jahres 1989 waren darauf gerichtet, den mit dem Gleichheitspostulat einherkommenden ,Wohlfahrtsdespotismus" (Alexis de Tocqueville) zu beseitigen und politische Freiheit zu erlangen. Der Freiheit wurde - wenngleich nur für kurze Zeit - der absolute Vorrang vor allen Einzelinteressen eingeräumt.

Dieser Vorrang der Freiheit ist allerdings sehr schnell, nicht nur in der ehemaligen DDR, der westlichen Konsumvariante des Gleichheitspostulats, Gleichheit durch Wohlstand, geopfert worden. Daß dabei ausgerechnet auf das Modell einer ungeregelten und durch gesetzliche Schranken nicht eingehegten Marktwirtschaft zurückgegriffen wurde, ist eine Ironie der Geschichte.

In der Praxis bedeutete diese Entscheidung massive soziale Verwerfungen und größere soziale Ungleichheit in Kauf zu nehmen, deren Ausmaß die Gleichheit politischer Teilnahmechancen ernsthaft gefährdet.

Da die normativen Grundlagen auf die sich die alte Gesellschaft berief, zerstört und moralisch diskreditiert sind, fällt es schwer, die entstandene Lücke auszufüllen. Das neue, schnell adaptierte Freiheitsideal entbehrt weitgehend der Dimension (sozialer) Gerechtigkeit. Auf die normative Grundfrage jeder demokratischen Gesellschaft: Freiheit in Verantwortung, gibt es keine erkennbaren Antworten.

Dieses Defizit wiegt um so schwerer, als der Zusammenbruch und Sturz der kommunistischen Systeme einen Großteil der Menschen in einer normativen und politisch-kulturellen Trümmerlandschaft zurückgelassen hat. Dies schafft keine günstige Voraussetzung für eine positive Identifikation mit demokratischen Ideen.

Ihre sozialen Erfahrungen haben die Menschen in einer Welt gesammelt, die symbolisch von der Verschmelzung von Gesellschaft und politischer Macht, de facto aber von der Monopolisierung des Politischen und der Instrumentalisierung jeglicher wirtschaftlicher, sozialer oder kultureller Aktivität durch die marxistisch-leninistische Partei gekennzeichnet war.

Die sozialen Beziehungen waren, wo sie über den Bereich des Privaten hinausreichten, mit dem umfassenden Gestaltungsanspruch der kommunistischen Partei konfrontiert. Jede Form eines 
Eigenlebens der Gesellschaft, gar der Anspruch auf autonome Interessenvertretung, waren ihr suspekt. Daher stehen heute - von den Resten der kleinen Bürgerrechtsgruppen abgesehen - keine sozialen Gruppierungen zur Verfügung, die über organisatorische Erfahrungen verfügten, welche für die Artikulation und Aggregation sozialer und politischer Interessen während der Transitionsperiode zu nutzen wären.

Die Atomisierung der Gesellschaft außerhalb der politisch strukturierten Sozialbeziehungen hinterließ eine amorphe Masse von Individuen, deren Demokratiefähigkeit - wenn überhaupt - nur über einen mühsamen und langwierigen Restrukturierungsprozeß sozialer Interessen und normativer Überzeugungen möglich ist. Dies stellt die Frage nach den Chancen eines Transfers von Loyalitäten zu einem demokratischen System. Die Chancen stehen nicht schlecht, wenn man im Anschluß an Robert A. Dahl vermutet, daß den konkurrierenden politischen Kräften daran gelegen sein muß, ein Mindestmaß an gegenseitiger Sicherheit zu schaffen, die es ihnen ermöglicht, einander zu tolerieren. ${ }^{7}$ Dazu bedarf es allerdings objektiver Bedingungen und bestimmter rechtlicher und institutioneller Voraussetzungen.

Selbst wenn diese gegeben sind, können das Ausmaß sozialer Ungleichheit, subkulturelle Cleavages oder bestimmte nationale Eigenschaften dazu beitragen, daß auftretende Konflikte nicht mit demokratischen Mitteln zu bearbeiten sind. Da die Demokratisierung neue Konflikte hervorbringen wird und in erster Instanz nicht mehr zu bieten hat als die Regulierung und Institutionalisierung ungewisser Ergebnisse und Entscheidungen, hängt viel von den ökonomischen und sozialen Rahmenbedingungen ab, unter denen sie stattfindet. ${ }^{8}$

\section{Demokratisierung und Re-Demokratisierung}

In solch unsicheren Konstellationen könnten demokratische Erfahrungen und Traditionen aus der Zeit vor der Diktatur nutzen, lägen sie nicht zu lange zurück. Gleichwohl ist zu fragen, ob es historische Traditionsbestände und Erfahrungen gibt, an die angeknüpft werden kann. 
Die gegenwärtigen Demokratisierungsprozesse haben eine Reihe von historischen Vorläufern. Eine Staatsbürgernation, die sich über die Sicherung bürgerlicher Freiheitsrechte und Verfahren der demokratischen Legitimation von Herrschaft definiert, hat allerdings in keinem der post-kommunistischen Länder eine solide Tradition - auch nicht im Osten Deutschlands.

Insoweit ist der Begriff „Re-Demokratisierung“ problematisch, es geht primär um Demokratisierung. Dies gilt es zu bedenken, wenn von der „Wiedergeburt" einer Bürgergesellschaft oder von civil society gesprochen wird. In den meisten Fällen handelt es sich um die Chance, sie erstmals zu verwirklichen.

Eine Bürgergesellschaft ist zudem nur eine von mehreren Möglichkeiten. Die bisherigen Erfahrungen der post-kommunistischen Länder zeigen, daß eine Vielfalt traditioneller politischkultureller Elemente nach dem Kollaps des Systems wieder ans Tageslicht kommen: nationale, regionale und/oder ethnische, religiöse und konfessionelle Identitäten, alte, vom Kommunismus verschüttete kulturelle Prägungen, nationale Rivalitäten, die Folgen diskriminierender Friedensschlüsse usw.

Bei der Suche nach Literatur zu historischen Vorbildern stößt man u. a. auf eine bei ihrem Erscheinen 1986 wenig beachtete und inzwischen berühmt gewordene vergleichende Studie über ,Transitions from Authoritarian Rule“. Dort hat Alfred Stepan in einem Beitrag verschiedene historische Wege zur Demokratisierung und Redemokratisierung herausgearbeitet, die sich drei Grundtypen zuordnen lassen:

1. Demokratisierung als Ergebnis von Kriegen und Eroberung;

2. Demokratisierung aus dem System heraus, d. h. angestoßen und vorangetrieben von reformbereiten Elitegruppen;

3. Demokratisierung aus der Gesellschaft heraus. ${ }^{9}$

Hierbei handelt es sich um Idealtypisierungen, die nur in einigen wenigen Fällen eine hohe Kongruenz mit empirischen Umbrüchen und Demokratisierungsprozessen aufweisen. Für die mittel-osteuropäischen Länder ist dieses Modell insoweit von Nutzen, als dort, in unterschiedlicher Intensität, alle drei Faktoren eine Rolle spielen.

Das erste Modell der Demokratisierung im Ergebnis von Kriegen und Eroberung bietet eine Parallele zum Ende des Kalten Krie- 
ges an. Ronald Steel argumentiert in einem Beitrag mit dem Titel „The End and the Beginning“ daß der Kalte Krieg zwar nicht den Untergang der Sowjetunion zum Ziel hatte, daß das Ende eines Imperiums aber normalerweise das Ergebnis einer katastrophalen Niederlage in einem Krieg sei.

Insofern ist der Ausgang des Kalten Krieges, der historisch einmalige, zumindest in der ersten Phase friedliche Zusammenbruch eines Imperiums durchaus mit dem Ende eines „klassischen“ Krieges zwischen Großmächten vergleichbar: einer der beiden hegemonialen Protagonisten existiert nicht mehr. ${ }^{10}$

Akzeptiert man diese Parallele, dann hält die historische Erfahrung nach 1945 drei verschiedene Formen der Demokratisierung und Re-Demokratisierung bereit: In den kleinen, von Deutschland überfallenen und besetzten Ländern Niederlande, Belgien, Norwegen und Dänemark wurden einstmals funktionierende Demokratien von innen wieder hergestellt, nachdem die Besatzung zu Ende war.

Demokratisierung unter Beobachtung und Anleitung von außen ist das Modell, nach dem in den Westzonen nach 1945 und der Bundesrepublik Deutschland nach 1949 verfahren wurde. Stepan ordnet auch - mit Abstrichen - Japan diesem Modell zu. Österreich, das ein Konkordanzmodell entwickelte, und Italien gehören partiell hierzu.

Den Erfolg des Demokratisierungsprozesses erklärt Stepan damit, daß die Grundlinien des Demokratiemodells eine große Konsistenz mit den sozialen und ökonomischen Mustern der Besatzungsmächte aufwiesen und der Prozeß von signifikanten sozialen und ökonomischen Reformen begleitet war. ${ }^{11}$

Hinzu kommt, daß heute in den post-kommunistischen Ländern die entscheidenden Elemente fehlen, die die Erfolgsgeschichte der Demokratien nach der Niederlage des Faschismus/Nationalsozialismus ausmachten: Die Möglichkeit und der Wille der „Sieger", sich massiv politisch, ökonomisch und militärisch in diesen Ländern zu engagieren und die neuentstehenden Demokratien zu garantieren. Dazu ist die Interessenlage zu diffus, es fehlt der eindeutig erkennbare Gegner. Die Situation ist durch Unübersichtlichkeit und sich überlagernde Interessen- und Konfliktkonstellationen gekennzeichnet, sowohl auf dem Felde der Innenpolitik als 
auch in der außen- und sicherheitspolitischen Arena. Hinzu kommt, daß sich die liberalen Demokratien selbst in keiner guten Verfassung befinden. Die Krisensymptome sind unübersehbar. Der entscheidende Unterschied zu den Jahren nach 1945 scheint mir zu sein, daß sich das demokratische Institutionensystem in den westlichen Ländern in einer Umstellungskrise befindet, also nicht erneut als selbstverständliches und unhinterfragtes Modell taugt, das nur übernommen werden muß.

\section{Institutionenbildung und Allokationsprobleme}

Diese Feststellung macht die 1989/1990 häufig geäußerte Einschätzung, daß es nur eine kurze Zeit brauche, um die nötigen institutionellen Strukturen und eine gefestigte bürgerliche Gesellschaft in Mittel-Osteuropa zu errichten, fraglich. Eine solche Erwartung erscheint aus heutiger Sicht übertrieben optimistisch. Die mentale und kulturelle Verankerung von Demokratie ist eine Sache von Generationen.

Die ersten Erfahrungen in den post-kommunistischen Ländern zeigen insoweit eine Gemeinsamkeit, als die Herstellung bestimmter Grundbedingungen, die das Funktionieren eines demokratischen Institutionensystems überhaupt erst ermöglichen, sich schwieriger gestaltet als erwartet.

Weder gibt es allgemein akzeptierte Vorstellungen über die wünschbare Struktur des Institutionensystems, noch über die Rolle, Kompetenzen und Befugnisse einzelner Institutionen.

Die Normenstruktur von Institutionen ist nicht durch Sozialisation internalisiert und produziert daher auch keine Regeln, die Handlungsgewohnheiten habitualisieren, stabilisieren und vereinheitlichen. Die Etablierung demokratischer Institutionen eröffnet zwar Möglichkeiten gemeinsamen Handelns, setzt aber voraus, daß den Institutionen ein Mindestmaß an Kompetenz zugetraut und ihnen ein gewisses $\mathrm{Ma} \beta$ an Vertrauen entgegengebracht wird.

Beides ist, wenn überhaupt, nur sehr beschränkt der Fall. Distanz und Mißtrauen gegenüber Institutionen beruhen auf den Erfahrungen mit dem alten System, auf problematischen und z. T. 
anarchischen Institutionalisierungsprozessen in der Zeit des Systemumbruchs und auf der Unkenntnis der rechtlichen und politischen Rahmenbedingungen des Handelns demokratischer Institutionen.

Zu dieser allgemeinen Distanz kommt in Ostdeutschland ein spezifisches Element: Die Institutionen werden als ,,von außen“ aufgezwungen erlebt oder interpretiert.

Der Umbau bzw. die Neugründung von Institutionen ist einer der wesentlichen Aspekte von Systemwechseln. Nach dem Ende von Diktaturen ist die bewußte Gestaltung institutioneller Zusammenhänge, die in der Lage sind, Konfliktaustragung zu ,verregeln" eine der zentralen Aufgaben, von deren erfolgreicher Bewältigung die Zukunft der Demokratie abhängt. Verregelung der Konfliktaustragung bedeutet sowohl Differenzierung des Institutionensystems als auch Entdifferenzierung und institutionelle Fusion. Dies schafft Allokationsprobleme.

Im Anschluß an M. Rainer Lepsius ${ }^{12}$ kann zwischen Kompetenzallokation, Ressourcenallokation, Legitimitätsallokation und Kontrollallokation unterschieden werden. Kompetenzallokation meint die Bestimmung und Verteilung von Entscheidungsrechten. Aus der Antwort auf die Frage, wer befugt ist zu entscheiden, ergeben sich die Handlungsspielräume einer Organisation, der Grad ihrer Autonomie und zugleich die Erwartungen, die an sie gerichtet werden. Die Kompetenzen der alten Institutionen waren total, die der neuen sind meist undeutlich ausgebildet. Ähnliches gilt für die Verteilung von Ressourcen.

Noch völlig offen ist die Frage nach der Rechtfertigung der neuen sozialen und politischen Ordnung. Da die gesamte Gesellschaft von neuen Herrschafts- und Abhängigkeitsverhältnissen durchzogen ist, bedarf es nicht nur legaler Verfahren, sondern nach allgemeiner Auffassung auch der Legitimitätsgeltung, um Akzeptanz zu erreichen.

Die Kontrollallokation schließlich regelt die Bestimmung und Verteilung der Anwendung von Sanktionen. Über Sanktionsmöglichkeiten verfügen nicht nur staatliche Institutionen, wie Polizei oder Gerichte, sondern auch Verbände, wie die Gewerkschaften (Streikrecht) oder Arbeitgeberverbände (Aussperrung). Kontrollfunktionen können z. B. von politischen Gruppierungen 
mit gesetzlich geregelten Beteiligungsformen oder durch die Mobilisierung der Institutionen öffentlicher Meinungsbildung wahrgenommen werden.

Differenzierte Sanktions- und Kontrollmöglichkeiten, ihre symmetrische oder asymmetrische Verteilung bestimmen wesentlich die inter-institutionellen Verflechtungen und bilden in liberalen Demokratien eine der Grundlagen für die korporative Verflechtung (Dahrendorf benutzt den Begriff „,korporatives Knäuel“") ${ }^{13}$ und für die Durchsetzungsfähigkeit von Institutionen im Herrschaftsgefüge einer Gesellschaft. Hier scheint das größte Defizit des sich herausbildenden neuen Institutionensystems zu liegen. Dies ist der Bereich, wo auf keinerlei Traditionslinien aus dem alten System und auf keine sozialen Erfahrungen zurückgegriffen werden kann.

Erschwerend kommt hinzu, daß die Transitionsgesellschaften vor dem Dilemma stehen, den Demokratisierungsprozeß in einer Zeit der tiefen ökonomischen Krise bewerkstelligen zu müssen, deren Ende nicht absehbar ist. Der ökonomische und politische Umbauprozeß ist mit erheblichen sozialen Kosten belastet.

Die entscheidende Frage ist, ob die Gesellschaften auf längere Zeit bereit sind, die Kosten des Transformationsprozesses zu tragen und ob die neu installierten demokratischen Regime die Fähigkeit haben werden, die daraus erwachsenden sozialen und politischen Konflikte mit demokratischen Mitteln zu bewältigen. Die ,worst case“ Antwort lautet, daß diejenigen, die von den sozialen Folgewirkungen der Transition am meisten betroffen sind, die neu installierten demokratischen Regeln nutzen könnten, die ökonomische Transformation zu blockieren und/oder die demokratische Herrschaft zu unterminieren. ${ }^{14}$ (Argumente hierfür bieten $u$. a. die Entwicklungen in Rußland, Litauen und Bulgarien.) Die schlechte Lage der Wirtschaft könnte diesen politischen Kräften - z. T. handelt es sich um Zweckbündnisse zwischen ehemaligen Kommunisten und Nationalpopulisten - die Argumente liefern.

Von daher ist bemerkenswert, was die Meinungsumfragen über die Einschätzung der wirtschaftlichen und politischen Situation sagen. Obwohl die gegenwärtige Situation negativ und die Funktionsfähigkeit der sozialistischen Wirtschaft in der Retrospektive 
häufig positiv beurteilt wird, ist die Erwartung in die positive Entwicklung der Volkswirtschaft in den nächsten Jahren unangebrochen. Der Grad des Optimismus korreliert unmittelbar mit der Beurteilung der früheren und gegenwärtigen wirtschaftlichen Situation. Wird die Leistungsfähigkeit der Planwirtschaft positiv beurteilt, fällt die Bewertung der gegenwärtigen und zukünftigen ökonomischen Entwicklung pessimistisch aus - und umgekehrt. ${ }^{15}$ Bemerkenswert ist, daß die Zufriedenheit oder Unzufriedenheit mit den neuen politischen Verhältnissen durchaus nicht mit der ökonomischen Situation der jeweiligen Länder und ihren Entwicklungsperspektiven korreliert. In den Ländern mit den besten Zukunftschancen - (Ostdeutschland, Tschechien, Polen und Ungarn) - sind nur die Tschechen mehrheitlich der Meinung, daß die Entwicklung im großen und ganzen in die richtige Richtung geht. Ausgerechnet die Albaner, aber auch die Bulgaren und Rumänen sehen zuversichtlich in die Zukunft, sind allerdings, ebenso, wie die übergroße Mehrheit der anderen Völker in Mittel-Osteuropa, unzufrieden mit dem Fortgang der Demokratisierung. ${ }^{16}$

Ich möchte es bei diesen Bemerkungen über die Bedeutung, der Relikte des alten Systems und die Chancen einer demokratischen Entwicklung belassen, und mich im folgenden der Frage zuwenden, welche Auswirkungen die Ereignisse des Jahres 1989 und die mühsamen Demokratisierungsprozesse in den post-kommunistischen Ländern auf die westlichen Demokratien, insbesondere die Bundesrepublik haben.

\section{Der Systemwechsel und die westlichen Demokratien}

Auch den westlichen Demokratien bereiten die Folgen von 1989 erhebliche Probleme. Ihnen ist eines der Grundaxiome ihres politischen Denkens und Handelns abhanden gekommen: die Bipolarität zwischen Ost und West. Die Ost-West-Auseinandersetzung wurde als dauerhafte, wenngleich sich in ihren Erscheinungsformen ändernde Konstante akzeptiert. In einem Buch über das Ende des Kalten Krieges argumentiert Ronald Steel, daß die Vorteile, die diese Situation für beide Hauptkontrahenten bereithielt, so manifest und die Kosten so berechenbar waren, daß es 
auf keiner Seite ein erkennbares Interesse gegeben habe, die Situation zu ändern. ${ }^{17}$ Daraus folge, daß die USA eine grundsätzliche Neubewertung ihrer Politik vornehmen müßten. Gleiches gilt für die Staaten der EG und Deutschland.

Neben der Notwendigkeit, die Interessenkalküle neu zu definieren, fordert das Ende des Kommunimus auch zu einer Neubewertung der normativen Grundlagen der politischen Ordnung heraus. Die revolutionären Umbrüche des Jahres 1989 haben das Ende des Sozialismus sowjetischen Typs und der Weltmacht Sowjetunion herbeigeführt. Damit wurde, um es mit Shlomo Avineri zu formulieren, ein ,Eckstein des westlichen demokratischen Ethos“ "beseitigt, nämlich „,die einhellige Verdammung von Regimen sowjetischen Typs. Ob der Sowjet-Kommunismus als Reich des Bösen betrachtet oder ob er nach dem Bild des gefallenen Engels interpretiert wurde: Er verdiente Ablehnung und mußte bekämpft werden. Heute gibt es diese Sichtweisen nicht mehr." ${ }^{\text {"18 }}$

Dies bedeutet, so meine These, daß die westlichen Demokratien von den Folgen des Endes des Kommunismus auch im Inneren, nicht nur in der Außen- und Sicherheitspolitik, erheblich tangiert sind.

Das Politikverständnis in den westlichen Demokratien, insbesondere in der alten Bundesrepublik, war nach dem II. Weltkrieg wesentlich von der Auseinandersetzung mit dem Kommunismus geprägt. Dies erklärt aber noch nicht, warum die ,,verordnete Demokratie" ${ }^{\text {"19 }}$ (um den von Theo Pirker eher pejorativ gemeinten Begriff positiv zu wenden) von den Bürgern angenommen wurde. Die Demokratie versprach eine stabile politische, ökonomische und soziale Ordnung nach westlichem Muster und Schutz vor der realen oder vermeintlichen Bedrohung durch den Kommunismus. Diese Einschätzung mindert nicht ihren Wert und ihre Erfolge, benennt aber zwei strukturelle Schwachstellen: Was geschieht, wenn diese beiden zentralen Legitimationsgrundlagen entfallen, wenn die ökonomischen Krisenerscheinungen das Versprechen sozialer Wohlfahrt gefährden und das integrierende Element der Abwehr gegen totalitäre Anfechtungen entfällt? Genau dies ist die politische Konstellation, in der sich die neue Bundesrepublik zu bewähren hat. 
Was bedeutet dies für die Zukunft der Demokratie? Hier bietet sich ein erneuter historischer Rückgriff an: Die modernen Massendemokratien erfuhren ihre erste Bewährungsprobe nach dem I. Weltkrieg in einer Zeit, als der europäische Kontinent den Zerfall der alten Imperien verarbeiten mußte, und als Kommunismus und Faschismus, später der Nationalsozialismus, mit allen Mitteln gegen die parlamentarischen Demokratien angingen, die oft nur schwach im Wertehaushalt der Bürger verankert waren.

Nach dem II. Weltkrieg wurde, ähnlich wie nach 1989, die Hoffnung auf eine neue Weltordnung sehr schnell zerstört. Die Auseinandersetzung der westlichen Demokratien, an ihrer Spitze die USA, mit dem Kommunismus dauerte fast ein halbes Jahrhundert. Innerhalb dieser Zeitspanne veränderte sich die Weltordnung fundamental - vor allem durch die Entkolonialisierung. Jedoch blieb die Konfrontation mit dem Kommunismus ein konstitutives Element des Selbstverständnisses und des Selbstbehauptungswillens liberaldemokratischer Systeme, auch dann noch, als Détente und Entspannungspolitik das Klima verbesserten.

Der Antikommunismus bildete den Kitt, der insbesondere die neuen Demokratien, die nach 1945 von außen implantiert worden waren, in ihrer Anfangsphase zusammenhielt. (Daß er, wie in der Mc Carthy-Ära in den USA zugleich auch eine ernsthafte Gefährdung freiheitlicher Demokratien von innen darstellen konnte, sei hier zumindest erwähnt.)

Dieses identitäts- und gemeinschaftsstiftende Element westlicher Demokratien ist entfallen, und es wird offenbar, daß sie erhebliche Probleme haben, sich auf eine in jeder Hinsicht multipolare Welt einzustellen.

Was bedeutet dies für die Bundesrepublik Deutschland? Es bedeutet vor allem, daß das normative Modell der wehrhaften Demokratie, das für die alte Bundesrepublik konstitutiv war, obsolet erscheint - zumindest seine Begründungszusammenhänge. Der Art. 20, Abs. 2 (alle Staatsgewalt geht vom Volke aus und wird vom Volk in Wahlen und Abstimmungen und durch besondere Organe der Gesetzgebung, der vollziehenden Gewalt und der Rechtssprechung ausgeübt), der Art. 20, Abs. 1, wonach die Bundesrepublik ein ,demokratischer und sozialer Bundesstaat“ und ist die „Ewigkeitsklausel“ des Art. 79, Abs. 3 verweisen zwar auf 
fundamentale Legitimationsgrundlagen der politischen und gesellschaftlichen Ordnung, sagen jedoch nichts Konkretes über das Demokratieverständnis des Grundgesetzgebers. Dies blieb dem Bundesverfassungsgericht vorbehalten. In seiner Definition der „freiheitlich demokratischen Grundordnung" hat es das demokratische Prinzip nicht positiv definiert, sondern in Abgrenzung zur antidemokratischen Herausforderung durch die ,Diktatur des Proletariats“ in der DDR und neo-nationalsozialistischen Auffassungen.

\section{Am Ende der alten Bundesrepublik}

Die Demokratie in Westdeutschland wurde in den letzten beiden Jahrzehnten von einem, in seinen Folgen noch immer nicht absehbaren Wandel des politischen Systems erfaßt. Dieser Wandel ist auf veränderte ökonomische und soziale Rahmenbedingungen und auf Veränderungen in den Wertorientierungen politisch organisations- und handlungsfähiger sozialer Gruppen zurückzuführen. Neue soziale Bewegungen und ein neuer Typ von Parteien beeinflußten das politische Geschehen und die Entwicklung des politischen Institutionensystems.

Die sozio-politischen Systeme des Westens waren durch wirtschaftliche Dynamik, schnell voranschreitende sozialstrukturelle Differenzierungsprozesse, Mobilität und wachsende Fragmentierung, die Auszehrung tradierter politisch-kultureller Milieus und deren Entkopplung (dealignment) von festen Parteibindungen gekennzeichnet. Tradierte Cleavagestrukturen wurden von einer Vielzahl von Interessengegensätzen abgelöst.

„Lebensweise“ und das Aufkommen neuer, normativ stark aufgeladener, sozialer und politischer Bewegungen hat in den letzten beiden Jahrzehnten zentrale Regeln demokratischer Systeme und die Rechtfertigungsfähigkeit der politischen Ordnung in Frage gestellt oder doch zumindest modifiziert. Legitimität geriet immer mehr in direkte Abhängigkeit von der subjektiven Wahrnehmung und Beurteilung der eigenen Lebenschancen und Lebensbedürfnisse. Daraus entstanden in Zeiten einer politischkulturellen Orientierungskrise Legitimitätszweifel, die mögli- 
cherweise tiefer gehen als die Folgen ökonomischer und sozialer Krisenfaktoren. ${ }^{21}$

Ganz anders in den post-kommunistischen Ländern einschließlich Ostdeutschlands. Dort reproduziert sich die alte Spaltung von nivellierter Sozialstruktur und hierarchischer Machtstruktur auf einer neuen Ebene. Die Gewinner des Systemwechsels waren in der Lage, die Chancen der Marktwirtschaft und der neuen institutionellen Strukturen zu nutzen, die große Mehrheit wird - vorerst und auf überschaubare Zeit - mit der Angst vor Arbeitslosigkeit und sozialem Abstieg und mit der Entwurzelung aus angestammten sozialen Milieus konfrontiert sein.

Der Individualisierung der Lebensstile im Westen kontrastiert scharf eine neue Form der Kollektivierung im Osten. Anstelle ideologisch motivierter und politisch durchgesetzter sozialer und kultureller Homogenisierung ist, in Folge der ökonomischen Krise, ein Prozeß der Entdifferenzierung und Nivellierung getreten. Lebensweltliche Orientierungen geraten unter massiven Druck der ökonomisch-sozialen Krise.

In Ostdeutschland kommt ein weiterer Faktor hinzu. Mit der staatlichen Einigung sind zwei völlig unterschiedlich strukturierte Gesellschaften aneinandergekoppelt worden: Die Gesellschaft der Bundesrepublik war durch die Ausdifferenzierung pluraler Lebenstile und neuer sozialer Milieus, soziale und regionale Mobilität und das Auseinanderdriften von Moderniserungsdynamik und Lebenswelt gekennzeichnet. In der Gesellschaft der DDR kontrastierte die nivellierte Sozialstruktur einer hierarchischen Machtstruktur.

Vieles spricht dafür, daß die Probleme, die aus dem Demokratisierungsprozeß in den post-kommunistischen Gesellschaften, einschließlich Ostdeutschlands, erwachsen, sich in Zukunft enger mit Krisenerscheinungen verknüpfen werden, die wir in den westlichen Demokratien beobachten. Die Bundesrepublik Deutschland bildet dabei ein besonders interessantes Beispiel, weil sie sich eine post-kommunistische Gesellschaft inkorporiert hat. Insofern steht sie vor einer doppelten Aufgabe: Sie muß die Umstellungsprobleme der politischen Institutionen der alten Bundesrepublik und die Anpassungskrise des ,,importierten“ Institutionensystems in den neuen Bundesländern bewältigen. 
Diese aktuellen Schwierigkeiten werden noch verstärkt durch die vielfältigen ungelösten Probleme aus den 80er Jahren. Bereits damals waren ökonomische, soziale und politische Krisensymptome erkennbar, die mit erheblichen sozialen Kosten verbunden waren. Die Stichworte lauteten: Dauerarbeitslosigkeit als Massenphänomen, Krisen in angestammten Schlüsselindustrien, ,Zweidrittelgesellschaft", Krise des Wohlfahrtsstaates, Ineffizienz staatlicher Interventionspolitik usw. ${ }^{22}$ Wolfgang Zapf hat diese komplexen Zusammenhänge als „Umstellungs- und Selektionskrise" gekennzeichnet. ${ }^{23}$

Beide sind noch nicht beendet. Die Umstellungskrise betrifft verschiedene Bereiche: Branchen und Regionen, das bestehende System der Arbeitsteilung, das durch einen neuen Schub technisch-technologischer Innovationen, vor allem im Bereich der Informations- und Produktionstechnologien umgestaltet wird, das Verhältnis von Arbeit und Freizeit, Erwerbs- und Familienarbeit, Veränderungen in der Gestaltung einzelner Lebensphasen (z. B. Hausfrauenrolle, Pensionierungsschock) und Lebensbereiche.

Die Selektionskrise zeigt sich vor allem in „Ungleichheiten in den verfügbaren Ressourcen und Kompetenzen, die im Wachstum verdeckt geblieben waren. ${ }^{\text {"24 }}$ Daraus entsteht, so Zapf, neue Segmentation, deren problematischstes Element die Dauerarbeitslosigkeit ist.

Diese ökonomischen und sozialen Krisenfaktoren schlugen in den Bereich des Politischen durch. Das korporativ-verbandliche Ordnungsmodell der Bundesrepublik geriet in die Diskussion. Dies Modell beruht auf der Anerkennung des Status und der Berechtigung des des Partners und einem faktischen Vertretungsmonopol organisierter Gruppen bei der Aushandlung von Entscheidungen und der Beilegung von Interessenkonflikten, und stellt damit einen Verhandlungsmodus zur Verfügung, der es ermöglicht, relativ dauerhafte und stabile Kompromisse zu erreichen, zu implementieren und in besonderer Weise die Interaktion und die Allokation von Ressourcen zwischen einer Gruppe privilegierter Akteure zu regeln.

Einige der kollektiven Akteure begannen bereits in den späten 80er Jahren, die Grundlagen dieses Modells in Frage zu stellen. 
Die Debatte über Deregulierung oder die wachsenden Schwierigkeiten der verbandlichen Partner, ihre Mitgliedschaft in Zeiten einer schnellen, krisenhaften Veränderung der ökonomischen und sozialen Rahmenbedingungen auf längerfristige strategische Ziele festzulegen ${ }^{25}$ stellen die Frage nach der zukünftigen Entwicklung des Systems organisierter Interessenvermittlung.

Gegenwärtig ist in der Bundesrepublik dieses über Jahrzehnte funktionierende, die ökonomische, soziale und politische Stabilität der alten Bundesrepublik wesentlich prägende Ordnungsmodell ernsthaft in Frage gestellt. Dies deutete sich bereits in der geringen Bereitschaft ostdeutscher und westdeutscher Investoren und Unternehmer an, sich den ostdeutschen Arbeitgeberverbänden anzuschließen und hat in der Auseinandersetzung in der ostdeutschen Metallindustrie um die vorzeitige Kündigung der geltenden Tarifverträge durch die Arbeitgeber einen ersten Höhepunkt erreicht.

Es besteht die Gefahr, daß die ökonomischen Krisenerscheinungen im Osten und die strukturelle Schwäche eines Akteurs, nämlich der Gewerkschaften, genutzt werden, um die Verregelung industrieller Arbeitsbeziehungen im Westen zu ändern.

Damit wäre aber mehr erreicht, als eine nachhaltige Schwächung der Gewerkschaften. Das korporative Interessenvermittlungssystem, in dem funktional differenzierte Verbände einen quasiöffentlichen Status und ein faktisches Vertretungsmonopol erhalten, wäre insgesamt in Frage gestellt.

Dies ist nur ein Bereich, neben anderen, in dem erkennbar wird, daß mit der staatlichen Einheit zwei Teilgesellschaften miteinander verbunden worden sind, in denen höchst unterschiedliche Erwartungen und Anforderungen an die Politik formuliert werden und der Prozeß der Interessenartikulation und -aggregation nach verschiedenen Mustern vor sich geht.

Während in der alten Bundesrepublik, wie in den meisten westlichen Industriegesellschaften, seit den frühen 70er Jahren neue Themen wie Umweltpolitik, Familienpolitik, Gleichberechtigung der Frau oder Rechte von Minderheiten eine erhebliche Rolle spielten und sich u. a. in der Neuformierung des Parteiensystems und der politischen Programmatik der Parteien niederschlugen, sind die Notwendigkeiten in Ostdeutschland, wie in den 
post-kommunistischen Ländern generell, eher denen der Nachkriegszeit vergleichbar: Auf der Tagesordnung stehen der wirtschaftliche Wiederaufbau, der Aufbau leistungsfähiger Institutionen und die Etablierung eines Systems sozialer Sicherung.

Dies bedeutet, daß in Ostdeutschland eher klassische Dimensionen wohlfahrtsstaatlicher Politik abgefordert werden, die sich, weder im parlamentarischen Raum, noch in den Parteien oder Interessenverbänden als anschlußfähig an die Interessenkonstellationen der alten Bundesrepublik erweisen.

Die Konfliktlinie zwischen ,,alter“ und „,neuer“ Politik, ${ }^{27}$ die sich durch die politische Auseinandersetzung der alten Bundesrepublik zog, hat eine zusätzliche regionale Dimension bekommen: In Ostdeutschland wird es für mindestens ein Jahrzehnt vor allem um ,,alte" Politik gehen.

Da dies etwa ein Fünftel der gesamtdeutschen Wählerschaft tangiert, sind die Rückwirkungen auf Politik, Programmatik und denkbare Koalitionsneigungen der Parteien unvermeidlich. Bereits jetzt sind die organisierten Interessengruppen unmittelbar davon betroffen. Sie müssen sowohl in ihren Binnen- als auch in ihren Außenbeziehungen zu den anderen Partnern des korporativen Gefüges auf ganz neue Art die Notwendigkeiten „klassischer" Wirtschaftspolitik und Wohlfahrtsstaatlichkeit mit subsidiärer Dezentralisierung und Deregulierung verbinden - eine kaum lösbare Aufgabe.

In einer Zeit, in der der wachsende Staatsinterventionismus selbst von den traditionellen Verfechtern des Sozialstaats als Problem erkannt wird, muß der Staat massiv (direkt oder indirekt) intervenieren, um die Folgen einer Deindustrialisierung und des $\mathrm{Zu}$ sammenbruchs der Agrarwirtschaft wenigstens einigermaßen aufzufangen.

Die Forderung nach staatlicher Intervention wird nicht unwesentlich durch alte Erwartungshaltungen und die Hoffnung auf die Steuerungsfähigkeit des Staates mitbestimmt. Der Effekt ist eine Ausweitung der staatlichen Agenda, die angesichts der wirtschaftlichen Rezession, der Verschuldung der öffentlichen Haushalte und der Notwendigkeit zu interner Umverteilung auch auf die Bereiche des Sozialstaats negativ zurückwirkt, die bislang relativ leistungsfähig waren. Damit entstehen und verfestigen sich 
bereits bestehende Konflikte, die erneut entlang der Ost-West-Linie angesiedelt sind.

Andererseits existiert für Ostdeutschland mit der alten Bundesrepublik ein Akteur, der - vergleichbar den westlichen Besatzungsmächten in der Nachkriegszeit - sowohl ein Demokratiemodell als auch erhebliche finanzielle Mittel und Personal zur Verfügung stellt, um den demokratischen Neuaufbau möglichst reibungslos zu gestalten.

Um die These zuzuspitzen: Die in Ostdeutschland häufig vertretene Meinung, die „Abwicklung“ der DDR sei der Entscheidung der ,Sieger“" zuzuschreiben, hat ihre Berechtigung, wenn man hinzufügt, daß diese Entscheidung auf dem in demokratischen Wahlen manifestierten Willen der Bevölkerung beruhte. Ironischerweise bot dieses „Siegerverhalten“ der Westdeutschen der ehemaligen DDR als einzigem post-kommunistischen Staat die Chance, den Transitionsprozeß in relativ kurzer Zeit hinter sich zu bringen: durch die Inkorporation in eine alles in allem funktionierende Demokratie und eine soziale Marktwirtschaft.

\section{Neukonstituierung der Bundesrepublik?}

Während die meisten anderen post-kommunistischen Gesellschaften noch mit dem ökonomischen Umbau, grundlegenden Verfassungsfragen, Institutionenbildung, der Nationenbildung und der Neuorientierung auf dem Felde der Außen- und Sicherheitspolitik beschäftigt sind, waren die Grundentscheidungen in Ostdeutschland zwischen November 1989 und dem 3. Oktober 1990 gefallen. Es sind die Folgen dieser Grundentscheidungen, wie z. B. der Wirtschafts-, Währungs- und Sozialunion, die die gegenwärtigen Probleme verursachen. Es führt kein Weg an der Einsicht vorbei, daß mit dem 3. Oktober 1990 nicht nur die DDR aufgehört hat zu existieren, sondern auch die alte Bundesrepublik Deutschland. Nichts Geringeres steht heute auf der Tagesordnung der Politik, als die Neukonstituierung des politischen Gemeinwesens der Bundesrepublik Deutschland.

Es geht, um eine Unterscheidung von David Easton aufzunehmen, um die Bestimmung der Grundzüge der neuen politischen 
Gemeinschaft und der neuen politischen Ordnung. In allen Transitionsgesellschaften steht neben der Institutionenbildung und der Rekonstruktion der Nationalstaaten die Vergewisserung über die normativen Grundlagen der neuen Gesellschaft auf der Tagesordnung. Dies geschieht gemeinhin, und auch in den anstehenden Debatten, im Rahmen einer Verfassungsdiskussion. Daher haben diese normativen Fragen zwangsläufig einen hohen Stellenwert.

Diese Argumentation bedeutet nicht, einem Gründungsmythos anheim zu fallen, wie er - insbesondere in der Linken - im Zusammenhang mit den Revolutionen von 1989 und ihren Folgen verbreitet war und z. T. noch ist. Die emphatische Beschwörung der verfassungsgebenden Gewalt des Volkes, des pouvoir constituante, die allen verfaßten Gewalten vorausgehe und diese erst hervorbringe, und der Verfassungsgebung als einem ,säkularreligiösen Akt der ,creatio ex nihilo “" 28 , trifft kaum den Kern der gegenwärtigen Probleme.

Mit Carl Joachim Friedrich kann dieser Argumentation entgegengehalten werden, daß die Vorstellung, die Gründung einer politischen Ordnung sei die Quelle aller echten Autorität, ${ }^{29}$ ihren Ursprung nicht in der Wirklichkeit hat. Sie wurzelt vielmehr in den Mythen, die sich um ein solches Gründungserlebnis ranken und in denen die Interessen, Werte und Überzeugungen der neuen Ordnung (aber wohl auch derer, die im Gründungsprozeß an den Rand gedrängt wurden) zum Ausdruck kommen. ,Es ist mit anderen Worten die neue Ordnung selbst und nicht deren Gründung, die die Grundlage der neuen Autorität bildet. ."

Friedrich führt die Schwierigkeiten, die in der Vergangenheit eine realistische Einschätzung des Gründungsprozesses politischer Ordnungen verhindert hätten darauf zurück, daß man zwischen politischer Gemeinschaft und politischer Ordnung nicht klar unterschieden habe.

„Politische Gemeinschaften werden nicht gegründet; sie bilden sich im Verlauf eines Prozesses ... allmählich heraus. Es ist allein die politische Ordnung einer solchen Gemeinschaft, die eines Gründungsaktes bedarf, oder, wie es in der Rechtssprache heißt ,instituiert" werden muß ... Revolutionen, die eine alte Ordnung zerstören, bilden daher zugleich auch den Auftakt für die Grün- 
dung einer neuen Ordnung; die Gemeinschaft dagegen kann zu einem großen Teil in ihrer alten Form weiterbestehen." Allerdings würde das Ausmaß an Veränderungen, die eine Revolution für die Gemeinschaft mit sich bringe, zugleich auch das Ausmaß an Neuerungen mitbestimmen, die für die neue politische Ordnung erforderlich seien.

Die Revolutionen von 1989 unterscheiden sich radikal von der amerikanischen oder der französischen Revolution, die zwar eine politische Ordnung stürzten, die soziale Gemeinschaft aber weitgehend unangetastet ließen. Ähnlich radikal wie in der russischen Revolution von 1917 - aber mit demokratischen Mitteln - wurde in den kommunistischen Ländern der alten politischen Gemeinschaft der Boden entzogen, einer Gemeinschaft, die auf der politischen Vergesellschaftung durch die marxistisch-leninistische Partei und dem Staatseigentum an den Produktionsmitteln beruhte. Was jedoch bleibt, sind die Wrackstücke dieser Gemeinschaft, „vagabundierende“" soziale Gruppen und Individuen, die ihren Ort in der Gesellschaft, der politischen Gemeinschaft und ihre Haltung zur politischen Ordnung neu bestimmen müssen. Das bedeutet, daß die politische Ordnung auf einer sehr schwachen, in eruptiver Bewegung befindlichen sozialen Gemeinschaft aufruht. Friedrich verweist darauf, daß die Gründung einer politischen Ordnung voraussetzt, daß die Bürger bereit sind, eine solche Gründung zu akzeptieren. ${ }^{32}$

Akzeptanz kann verschieden motiviert sein. Sie kann bedeuten, aus Opportunimus oder Einsicht, sich in die Verhältnisse zu fügen, ihr kann aber auch ein sozialer und politischer Lern- und Adaptionsprozeß zugrunde liegen, oder sie kann das Ergebnis normativer Übereinstimmung mit den Werten und Zielen einer politischen Ordnung bedeuten.

Die Funktionsfähigkeit freiheitlicher Demokratien basiert wesentlich auf ihren verfassungsmäßigen Grundlagen, auf dem in Verfassungen normierten institutionellen Gefüge und auf den dort kodifizierten Verfahren. Aber sie beruht auch auf der sozialen Gestalt der Gesellschaftsordnung und den Werten und Normen, denen sich die Bürger verpflichtet fühlen. Diese normative Identifizierung mit der politischen Ordnung wird im allgemeinen als Grundlage ihrer Legitimität angesehen. Da in einer säkularisier- 
ten Gesellschaft die Legitimationsgrundlagen von Macht und Herrschaft wesentlich vom Handeln der politischen Akteure und der Leistungsfähigkeit der Institutionen abhängen, gibt es keine Garantie dafür, daß politische Institutionen auch bei radikalen Veränderungen, wie sie z. B. fundamentale Wechsel in den Wertehaltungen der Bürger darstellen, oder wie sie im Zuge der Inkorporation eines anderen Gesellschaftstypus in die eigene Gesellschaft auftreten, ihren Legitimitätsanspruch bewahren können. ${ }^{33}$ Selbst das erforderliche Mindestmaß an Loyalität ist nicht selbstverständlich gesichert. ${ }^{34}$

Legitimität ist in den Transitionsgesellschaften noch nicht vorhanden, selbst die Loyalität ist gefährdet. Was es allenfalls gibt, ist „compliance“, im Sinne eines fügsamem Einverständnisses mit der veränderten Situation.

Guiseppe di Palma spricht in einem Essay über die demokratischen Transitionen davon, daß eine breite Konversion (,widespread popular conversion“"), ${ }^{35}$ genüge, um die Transition zur Demokratie einigermaßen zu sichern. Ein minimalistischer Blick auf das Problem der Legitimität sei ausreichend. Transitionen vom Kommunismus seien ,transitions from the top“. Dies sei mehr als plausibel, wenn man die Rolle bedenke, die die regierenden Parteien im alten System gespielt haben. Von daher sei nicht zu erwarten, daß breite Schichten der Bevölkerung mit den Werten und Zielen der Demokratie vertraut seien. Allerdings seien die aus der Herrschaft der Kommunisten erwachsenen kulturellen Hindernisse auch nicht unüberwindbar: Die ehemaligen kommunistischen Eliten seien möglicherweise nicht „resozialisierbar“, ihr Eigeninteresse gebiete aber eine Anpassung an die neuen Verhältnisse. Einer der entscheidenden inneren Faktoren sei das Ausmaß, in dem die Bürger über Dekaden der kulturellen Isolation und sozialistischen Sozialisation widerstanden hätten und nun von westlichem Lebensstil und westlichen Wertvorstellungen fasziniert seien.

$\mathrm{Da}$ es den Kommunisten, anders als vielen Rechts-Diktaturen nicht gelungen sei, Formen einer semipluralistischen Kooperation zwischen Regime und Gesellschaft zustandezubringen, sei jetzt eine veritable massenhafte Konversion zur Demokratie zu beobachten. Dies genüge als Legitimationsgrundlage. ${ }^{36}$ 
Ist es nicht gleichwohl besser, wenn die neuen Demokratien auf der Legitimitätsgeltung durch ihre Bürger beruhen? Guiseppe di Palma verneint: Legitimität in ihrer anspruchsvolleren Bedeutung sei ebenso rar, wie unnötig. Auch ohne sie seien die neuen Demokratien nicht ernsthaft gefährdet, da jede attraktive Alternative fehle und das Maß der Erwartungen darauf, was die Demokratie erreichen könne und wolle, steige. ${ }^{37}$ Diese Einschätzung aus dem Jahre $1990 \mathrm{mu}$ wohl inzwischen modifiziert werden. Gegen diese Argumentationsfigur, die den Charme eines gewissen Pragmatismus für sich hat - hinter dem sich ihr unterliegender normativer Gehalt (Demokratie als unausweichliches Ergebnis) verbirgt - ist vor allem einzuwenden, daß mit einer „Konversion“ alter Führungsgruppen zur Demokratie und mit der Akzeptanz formaler Verfahren, die für das Funktionieren und die Überlebensfähigkeit von Demokratien unabdingbaren allgemein akzeptierten Wertorientierungen und Verhaltensmuster nicht mitgeliefert werden.

Die Erfahrungen mit Demokratisierungsprozessen nach dem II. Weltkrieg zeigen, daß dies ein langwieriger Prozeß ist, der jedoch erheblich erleichtert wird, wenn die neue politische Ordnung auf einer bewußten Wertentscheidung der Bürger gründet. Dazu aber bedarf es sozialer und kultureller Rahmenbedingungen, die eine solche Wertentscheidung erst ermöglichen. Und es bedarf des erkennbaren politischen Willens, eine Konversion zur Demokratie zu akzeptieren und zu honorieren. Bisher hat die Bundesrepublik noch nicht erkennen lassen, daß sie zu einer ähnlichen sozialen und politischen Integrationsleistung wie nach 1945 bereit ist. Davon wird entscheidend abhängen, wie die Demokratie in der neuen Bundesrepublik gesellschaftlich verankert sein wird.

Der Historiker und Altphilologe Donald Kagan vertritt die Auffassung, daß der dauerhafte Erfolg der Demokratie von drei Bedingungen abhängig ist:

„Erstens braucht es dazu eine Reihe funktionierender Institutionen, zweitens eine Bürgerschaft, die über Einsicht in die Prinzipien der Demokratie verfügt oder jedenfalls einen der demokratischen Lebensweise entsprechenden Charakter ausgebildet hat, und drittens eine qualifizierte Führung, zumindest in kritischen Augenblicken. Gelegentlich ist diese dritte Bedingung die wich- 
tigste und kann sogar Schwächen bei den anderen beiden Bedingungen wettmachen. “38

Die gegenwärtige Konstellation ist dadurch gekennzeichnet, daß das Institutionensystem an Leistungsdefiziten leidet und daß es für die Probleme der Transitionsgesellschaft im Osten Deutschlands nur bedingt anschlußfähig ist. Die Bereitschaft der Bürger, sich im Rahmen verfaßter Beteiligungsformen zu aktivieren, sinkt. Es ist ein postdiktatorischer Antiinstitutionen-, Antiparteien- und Antimobilisierungseffekt zu beobachten, der noch durch die geringe Bereitschaft im Westen, spezifische Erfahrungen und Verhaltensweisen aus dem Osten zu akzeptieren, verstärkt wird. Die politischen Führungsschichten schließlich verhalten sich wie in einem Filmtitel von Alexander Kluge: ,Die Artisten in der Zirkuskuppel - ratlos".

Demokratie nach dem Ende des Kommunismus? Das Fazit bleibt, wie könnte es anders sein, zwiespältig. Ich schlage vor, dem Rat von Gabriel A. Almond zu folgen, der Ende der 60er Jahre meinte, die Politikwissenschaftler möchten sich, wenn sie ,die Vielfalt und Instabilität der heutigen politischen Systeme betrachten, - getreu der Tradition der Aufklärung - ihre Hoffnung auf den allgemeinen Sieg der Demokratie bewahren; wir können aber wohl kaum annehmen, daß dieses Ziel in absehbarer Zeit erreicht sein wird. “39 


\section{Anmerkungen}

1 Shlomo Avineri, Eine neue Welt - oder die Wiederkehr der alten? Über Postkommunismus, Nationalismus und das vermeintliche „Ende der Geschichte“, in: Frankfurter Allgemeine Zeitung vom 2. Februar 1993, S. 10.

2 Vgl.: Dietrich Rueschemeyer/Evelyne Huber Stephens/John D. Stephens, Capitalist Development and Democracy, Chicago: University of Chicago Press 1992, S. VII.

3 Alain Minc, La vengeance des nation, Paris: Bernard Grasset 1990; (dt.: Die Wiedergeburt des Nationalismus in Europa, Hamburg: Hoffmann und Campe 1992).

4 Alain Minc, Die Wiedergeburt, a. a. O., S. 57 f.

5 Vgl.: hierzu: RalfDahrendorf, Betrachtungen über die Revolution in Europa in einem Brief, der an einen Herrn in Warschau gerichtet ist 1990, Stuttgart: DVA 1990, S. 76.

6 Octavio Paz, Wir müssen keine Brüder sein. Über das Zeitalter der Entdeckungen und die Zukunft der Demokratie, in: Frankfurter Allgemeine Zeitung vom 2. Oktober 1992, Beilage „Bilder und Zeiten“.

7 Vgl.: Robert A. Dahl, Polyarchy: Participation and Opposition, New Haven: Yale University Press 1971, S. 16.

8 Guiseppe di Palma, To Craft Democracies. An Essay on Democratic Transitions, Berkeley/Los Angeles/Oxford: University of California Press 1990, S. 28.

9 Vgl.: Alfred Stepan, in: Guillermo O'Donnel/Philippe C. Schmitter/Laurence Whitehead (Eds.), Transitions from Authoritarian Rule. Comparative Perspectives, Baltimore/London: The Johns Hopkins University Press 1986, S. 64-84.

10 Vgl.: Ronald Steel, The End and the Beginning, in; Michael J. Hogan (Ed.), The End of the Cold War. Its Meanings and Implications, Cambridge, Mass.: Cambridge University Press 1992, S. 104. (Steel ist Professor for international relations an der University of Southern California)

11 Eine solche Konstellation kann nach Auffassung von Stepan, formuliert im Jahre 1986, praktisch kaum noch einmal eintreten, da es, selbst nach einem Krieg, keine hegemoniale Macht mehr geben werde, wie die USA 1945, sich eine vergleichbare Situation also in einem wesentlich komplexeren Set von Faktoren bewegen werde. Vgl.: Stepan, Paths. a. a. O., $\mathrm{S} .71 \mathrm{f}$.

12 M. Rainer Lepsius, Interessen, Ideen und Institutionen, Opladen: Westdeutscher Verlag 1990. 
13 Dahrendorf, Die Revolution, a.a.O., S. 130.

14 Vgl.: Lászlo Bruszt, Transformative Politics: Social Costs and Social Peace in East Central Europe, in: East European Politics and Societies, Vol 6 (Winter 1992), No. 1, S. 55-72.

$15 \mathrm{Vgl}$. hierzu: Demokratie und Martkwirtschaft in neuen Demokratien. Veränderungen im Meinungsklima in: Bjelorußland, Bulgarien, Kroatien, Polen, Rumänien, Slowakei, Slowenien, Tschechien, Ukraine, Ungarn (Neue Demokratien-Barometer, Dezember 1992), Paul Lazarsfeld Gesellschaft für Sozialforschung, Wien.

16 Hungary: Residents Feel Discontented - But They're Staying Just the Same, in: The Hungarian Obeserver,Vol.6, No.4 (April 1993), S.11-13.

17 Steel, The End, a.a.O., S. 104.

18 Avineri, Eine neue Welt, a.a.O.

19 Vgl.: Theo Pirker, Die verordnete Demokratie. Grundlagen und Erscheinungen der „Restauration“, Berlin: Olle und Wolter 1977.

20 Vgl.: BVerfGE Bd. 5, 85/133 ff. (KPD-Urteil und BVerfGE Bd. 2, 1/10 ff. (SRP-Urteil); vgl. dazu ferner: Eberhard Denninger (Hrsg.), Freiheitlich demokratische Grundordnung, 2 Bde., Frankfurt/M.: 1976; Maunz/Dürig/Herzog/Scholz, Kommentar zum Grundgesetz, München 1984.

21 Vgl.: Bernd Guggenberger/Claus Offe, An den Grenzen der Mehrheitsdemokratie. Opladen: Westdeutscher Verlag 1984.

22 Vgl. hierzu: David Held, Models of Democracy, Stanford: Stanford University Press 1987, S. 231.

23 Wolfgang Zapf, Zur Diskussion um Krise und Innovationschancen in westlichen Demokratien, in: Max Kaase (Hrsg.) Politische Wissenschaft und politische Ordnung. Analysen zu Theorie und Empirie demokratischer Regierungsweise Opladen: Westdeutscher Verlag 1986, S. 52-60.

24 Zapf, Zur Diskussion a. a. O., S. 53.

25 Vgl.: Wolfgang Streeck/Philippe C. Schmitter, Gemeinschaft, Markt und Staat - und die Verbände? Der mögliche Beitrag Interessenorganisationen zur sozialen Ordnung, in: Journal für Sozialforschung, 25. Jg. (1985), Nr. 2, S. 133-157.

26 Vgl. hierzu: Werner Reutter, Korporatismustheorien. Kritik, Vergleich, Perspektiven, Frankfurt a. M./ u. a.: Peter Lang 1991.

27 Vgl.: Russel Dalton/Scott C. Flanagan/ Paul Allen Beck (Hrsg.) Electoral Change in Advanced Industrial Democracies: Realignment or Dealignment?, Princeton: Princeton University Press 1984.

28 Ulrich K. Preuß, Das Recht der Revolution. Über das Eindringen der Ethik in die Politik, in: Frankfurter Allgemeine Zeitung vom 29. August 1992, Beilage „Bilder und Zeiten“; Die „Kronzeugen“ dieser Argu- 
mentationsfigur sind Hannah Arendt, Cornelius Castoriadis und Claude Lefort. Vgl.: Ulrich Rödel/Günter Frankenberg/Helmut Dubiel, Die demokratische Frage, Frankfurt/M.: Suhrkamp 1989; Hannah Arendt, Über die Revolution, München: Piper 1974; Cornelius Castoriadis, Gesellschaft als imaginäre Institution, Frankfurt a. M.: Suhrkamp 1984; Claude Lefort, Eléments d'une critique de la bureaucratie, 2. Aufl., Paris 1979; ders., L'invention démocratique, Paris 1981.

29 Friedrich argumentiert hier gegen Hannah Arendt; vgl.: Carl Joachim Friedrich, Politik als Prozeß der Gemeinschaftsbildung. Eine empirische Theorie, Köln/Opladen: Westdeutscher Verlag 1970, S. 258.

30 Friedrich, Politik, a. a. O., S. 258.

31 Friedrich, Politik, a. a. o., S. 259.

32 Friedrich, Politik, a. a. O., S. 261; Friedrich exemplifiziert diese Zusammenhänge an drei Gründungen nach dem II. Weltkrieg: des Staates Israel 1948, der Bundesrepublik Deutschland 1949 und des „Commonwealth of Puerto Rico" 1952.

33 Vgl. hierzu: Ulrich Rödel/Günter Frankenberg/Helmut Dubiel, Die demokratische Frage. Ein Essay, Frankfurt/M.: Suhrkamp 1989, S. 20 ff.

34 Zum Problem von Legitimität und Loyalität als „Erbe“ des alten Systems vgl.: Sigrid Meuschel, Legitimation und Parteiherrschaft in der DDR. Zum Paradox von Stabilität und Revolution in der DDR 1945 1989, Frankfurt/M.: Suhrkamp 1992.

35 di Palma, To Craft, a. a. O., S. 146.

36 di Palma, To Craft, a. a. O., S. 148.

37 di Palma, To Craft, a. a. O., S. 149.

38 Donald Kagan, Ein delikates Gewächs, in: Deutsches Allgemeines Sonntagsblatt vom 21. August 1992, S. 10.

39 Gabriel A. Almond, Politische Systeme und politischer Wandel, in: Wolfgang Zapf (Hrsg.), Theorien des sozialen Wandels, Köln/Berlin: Kiepenheuer 1969, S. 212. 


\section{Gert-Joachim Glaeßner}

Geboren 1944 in Erfurt.

Studium der Politologie, Soziologie und Volkswirtschaftslehre. 1976 Promotion zum Dr. rer. pol. 1982 Habilitation für das Fach Politische Wissenschaft. 1986 - 1992 Professor für Politische Wissenschaft an der Freien Universität Berlin.

Gastprofessuren an Universitäten in den USA und Großbritannien.

Seit 1992 Professor für Politische Wissenschaft (Lehrstuhl Innenpolitik) am Fachbereich Sozialwissenschaften der HumboldtUniversität zu Berlin.

Wichtigste Veröffentlichungen

The Unification Process in Germany. From Dictatorship to Democracy, London (Pinter) 1992

Der schwierige Weg zur Demokratie. Vom Ende der DDR zur deutschen Einheit, Opladen: Westdeutscher Verlag 1991; (2. Aufl. 1992); (japanische Übers. 1993)

Arbeiterbewegung und Genossenschaft. Entstehung und Entwicklung der Konsumgenossenschaften am Beispiel Berlins, Göttingen/Zürich: Vandenhoeck \& Ruprecht (Marburger Schriften zum Genossenschaftswesen) 1989

Die andere deutsche Republik. Gesellschaft und Politik in der DDR, Opladen: Westdeutscher Verlag 1989

Der lange Weg zur Einheit. Studien zum Transformationsprozeß in Ostdeutschland (Hrsg.), Berlin: Dietz 1993

The German Revolution 1989. Causes and Consequences (zusammen mit Ian Wallace), (Hrsg.), Oxford: Berg Publisher 1992 Eine deutsche Revolution. Der Umbruch in der DDR, seine Ur- 
sachen und Folgen (Hrsg.), Frankfurt/M.: Peter Lang Verlag 1991; (2. Aufl. 1992)

Das Ende eines Experiments. Umbruch in der DDR und deutsche Einheit (Hrsg. zusammen mit Rolf Reißig), Berlin: Dietz Verlag 1991

Die politischen Systeme der sozialistischen Länder. (Hrsg. zusammen mit Michael Reimann), Frankfurt/M.: Peter Lang Verlag 1991 
In der Reihe Öffentliche Vorlesungen sind erschienen:

$1 \quad$ Volker Gerhardt

Zur philosophischen Tradition der HumboldtUniversität

2 Hasso Hofmann

Die versprochene Menschenwürde

3 Heinrich August Winkler Von Hitler zu Weimar

Die Arbeiterbewegung und das Scheitern der ersten deutschen Demokratie

$4 \quad$ Michael Borgolte

„Totale Geschichte“ des Mittelalters?

Das Beispiel der Stiftungen

$5 \quad$ Wilfried Nippel

Max Weber und die Althistorie seiner Zeit

$6 \quad$ Heinz Schilling

Am Anfang waren Luther, Loyola und Calvin ein religionssoziologisch-entwicklungs-

geschichtlicher Vergleich

$7 \quad$ Hartmut Harnisch

Adel und Großgrundbesitz im ostelbischen

Preußen 1800 - 1914 


\section{Fritz Jost}

Selbststeuerung des Justizsystems durch richterliche Ordnungen

9 Erwin J. Haeberle

Berlin und die internationale Sexualwissenschaft

10 Herbert Schnädelbach

Hegels Lehre von der Wahrheit

11 Felix Herzog

Über die Grenzen der Wirksamkeit des

Strafrechts

12 Hans-Peter Müller

Soziale Differenzierung und Individualität

Georg Simmels Gesellschafts- und Zeitdiagnose

13 Thomas Raiser

Aufgaben der Rechtssoziologie als Zweig der

Rechtswissenschaft

14 Ludolf Herbst

Der Marshallplan als Herrschaftsinstrument?

Überlegungen zur Struktur amerikanischer Nach-

kriegspolitik 
Es erscheinen demnächst:

16 Arndt Sorge

Arbeit, Organisation und Arbeitsbeziehungen

in Ostdeutschland

17 Achim Leube

Semnonen, Burgunden, Alamannen

Archäologische Beiträge zur germanischen Früh-

geschichte

18 Klaus-Peter Johne

Von der Kolonenwirtschaft zum Kolonat

Ein römisches Abhängigkeitsverhältnis im Spiegel der

Forschung

19 Volker Gerhard

Die Politik und das Leben

20 Clemens Wurm

Großbritannien, Frankreich und die

westeuropäische Integration 